\title{
Malignant Glomus Tumor of the Heart in a 64-Year-Old Male Presenting with Stroke
}

Othaniel Philip Balisan, C Philip Teomar Radin II, Randell Arias, Felipe Templo, Jr.

\section{ABSTRACT}

Glomus tumor is a soft tissue neoplasm usually observed as a solitary, or sometimes multicentric painful mass, that rarely occurs extracutaneously. We describe a rare case of malignant glomus tumor of the heart in a 64-year-old male diagnosed with a left ventricular mass. Echocardiography and color flow Doppler revealed a large echogenic mobile structure in the left ventricular cavity that was surgically resected. The histopathologic diagnosis was malignant cardiac glomus tumor. We describe the histopathology, differential diagnosis and clinical presentation of this extremely rare primary cardiac tumor.

Key words: cardiac tumor, left ventricular mass, glomus tumor, malignant cardiac glomangioma, embolic stroke

ISSN 2507-8364 (Online)

Printed in the Philippines.

Copyright $(2018$ by the PJP.

Received: 3 November 2017.

Accepted: 19 February 2018.

Published online first: 23 February 2018.

https://doi.org/10.21141/PJP.2018.005

Corresponding author: Othaniel Philip R. Balisan, MD

E-mail:phcpathology@yahoo.com

\section{INTRODUCTION}

Glomus tumor is a mesenchymal neoplasm involving less than $2 \%$ of soft tissue tumors. ${ }^{1}$ They usually present as painful solitary or multicentric masses, most commonly located subungually and also occurs in the dermis or subcutis of the upper or lower extremity. ${ }^{2}$ They are reported to also primarily arise in unusual locations such as the respiratory tract, ${ }^{3}$ mediastinum, ${ }^{4}$ stomach, ${ }^{5}$ vulva, ${ }^{6}$ penis, ${ }^{7}$ and eye. ${ }^{8}$

\section{CASE}

The patient is a 64-year-old male who presented with right-sided body weakness but without episodes of chest pain, dyspnea, orthopnea or palpitations. Consultation was done and he was advised surgical intervention, hence admitted in our institution. During his hospital stay, computed tomography (CT) scan of the head was done revealing finding of acute infarct in the right thalamus and right hippocampus, and old hemorrhagic infarct in the right frontal lobe and bilateral occipital lobes (Figure 1).

Echocardiography and color flow Doppler reveal a large echogenic mobile structure seen in the left ventricular cavity, which appears to have a stalk attached to the anterior interventricular septum and anterolateral wall. This measures $3.4 \times 2.4 \mathrm{~cm}$ at its widest dimension with an estimated area of $6.8 \mathrm{~cm}$. Excision of the mass was done. The specimen was sent for histopathologic examination. The procedure was uneventful and patient was eventually discharged improved.

On gross examination, the specimen consists of several fragmented, tan-brown to gray, irregular, rubbery to friable tissues aggregately measuring $3 \times 2 \times 1.5 \mathrm{~cm}$ (Figure 2). Microscopic sections revealed soft tissue fragments composed of lesional cells in sheets surrounding endothelial-cell-lined vascular channels and supported by fibrous stroma. The individual lesional cells exhibit round to ovoid, hyperchromatic to vesicular nuclei, some with prominent nucleoli and lightly eosinophilic cytoplasm. Few mitotic figures are appreciated. Immunohistochemical studies show the lesional cells with diffuse cytoplasmic reactivity against vimentin and focal reactivity against smooth muscle actin and pancytokeratin (AE1/3). S-100, desmin, calretinin and chromogranin are all negative. The Ki-67 proliferation fraction is 

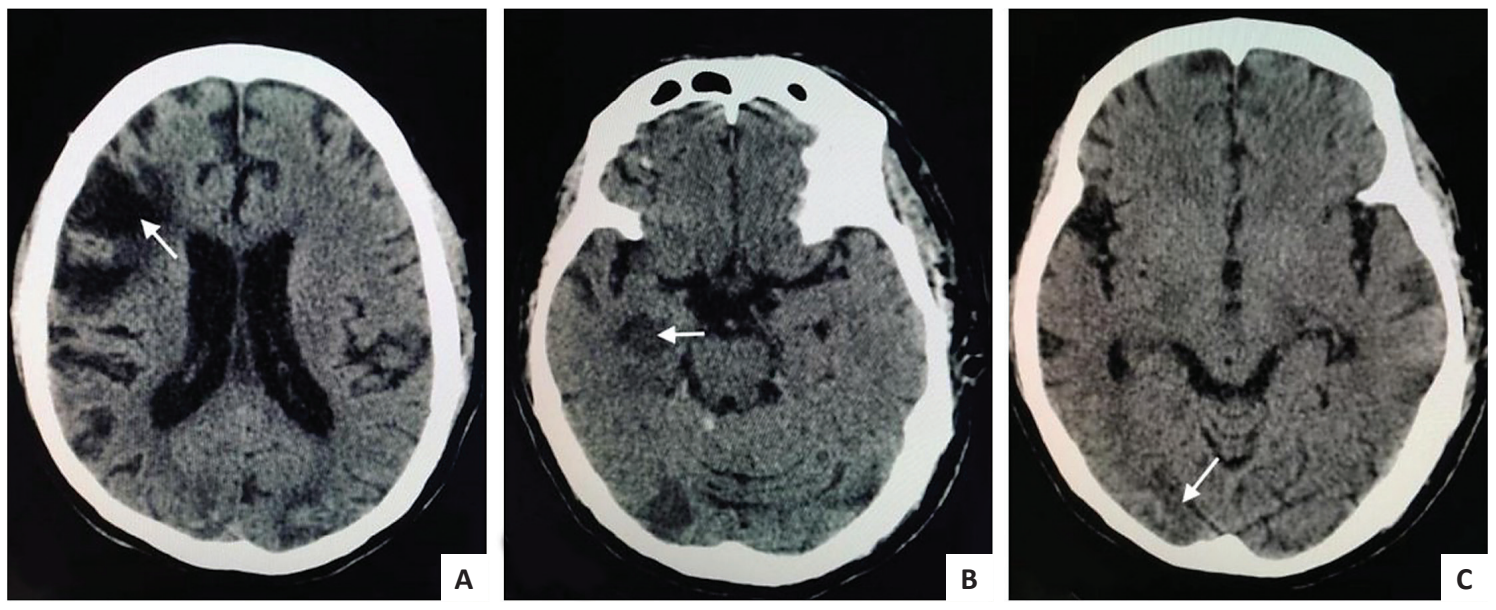

Figure 1. (A) CT scan of the head showing encephalomalacic changes in the right frontal lobe. (B) Acute infarct involving the right hippocampus. (C) Hemorrhagic infarct in the occipital lobe.

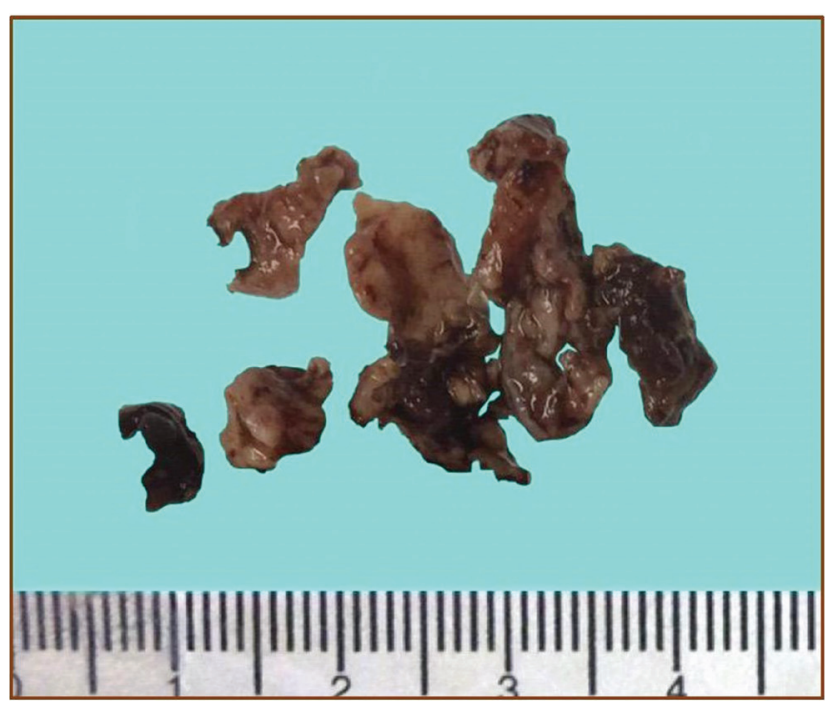

Figure 2. Gross features of the neoplasm showing several fragments of tan-brown, friable to rubbery tissue.

between $20-25 \%$ (Figures 3). These findings, in the context of the light microscopic findings provided further evidence to support the above interpretations.

\section{DISCUSSION}

Glomus tumors are rare neoplasms comprising of less than $2 \%$ of all soft tissue tumors. ${ }^{1}$ These arise from a neuromyoarterial structure, the glomus body, ${ }^{3}$ a thermoregulator usually found in dermal and precoccygeal areas, ${ }^{1}$ whose main function is to regulate skin circulation. ${ }^{3}$ These may also be localized subungually involving the finger tip pulp, which accounts to the common presentation of the tumor as subungual lesion of the fingers, ${ }^{3}$ but may also involve areas of the deep dermis in the distal extremities including the palm, wrist, forearm, and foot. ${ }^{1}$ Most glomus tumors are solitary, but may appear as multicentric. ${ }^{2}$ They often occur in children to adolescent age with a predilection toward males. Multicentric tumors, however, are more prevalent in females. ${ }^{7}$ They may appear asymptomatic ${ }^{7}$ but typically presents with a characteristic clinical triad of pain, pinpoint tenderness and cold hypersensitivity. ${ }^{4}$ Glomus tumors have also been reported in rare locations such as the respiratory tract, ${ }^{3}$ mediastinum, ${ }^{4}$ stomach, ${ }^{5}$ vulva, ${ }^{6}$ penis $^{7}$ and eye. ${ }^{8}$ We describe a highly unusual case of a glomus tumor of the heart. To our knowledge, this is the fourth glomus tumor case to be reported originating from the heart, and a first in this institution. ${ }^{9-11}$

Typical glomus tumors are well-circumscribed lesions composed of capillary-sized vessels enclosed by cuffs of glomus cells. ${ }^{7}$ These are subdivided into three categories; solid glomus tumor, glomangioma, or glomangiomyoma, which is dependent upon the composition of vascular structures, smooth muscle cells, glomus cells and their varying proportions. ${ }^{3}$ Characteristic cytomorphologic features are small, uniform, and round cells with centrally located, round nuclei, rimmed by an amphophilic to lightly eosinophilic cytoplasm. The solid glomus tumor is the most common involving approximately $75 \%$ of cases, glomangiomas being the second most common involving approximately $20 \%$, and lastly glomangiomyomas accounting only $<5 \%$ of cases. ${ }^{3}$ Atypical glomus tumors are classified by tumor location, size, nuclear atypia, and mitotic figures. This can be categorized into four groups; malignant glomus tumor, glomus tumor of uncertain malignant potential, symplastic glomus tumor, and glomangiomatosis. ${ }^{4}$ Malignant glomus tumors account to less than 20 cases $^{2}$ and shows features of severe atypia, increased mitotic activity (>5/50 hpf) or presence of atypical mitosis. Deeply located glomus tumors, located in the visceral organs, including the heart, falls under malignant glomus tumor or glomus tumor of uncertain malignant potential. ${ }^{4}$ Folpe et al., proposed a reclassification of malignant glomus tumor to include deep location and a size of more than 2 $\mathrm{cm}$ in addition to increased mitotic activity and nuclear atypia in its criteria. ${ }^{12}$ Glomus tumor of uncertain malignant potential may only have one of the following: superficial location with increased mitotic activity $(>5 / 50 \mathrm{hpf}$ ), or a size of $>2 \mathrm{~cm}$ only, or deep location., ${ }^{4}$ Tumors with severe nuclear atypia but lacks criteria for malignant glomus tumor are classified under symplastic glomus tumor. Tumors with diffuse growth resembling angiomatosis with prominent glomus component but lacks criteria for malignant glomus tumor or glomus tumor of uncertain malignant potential are classified under glomangiomatosis. ${ }^{4}$

Approximately 10\% of glomus tumors exist as multiple variants with hereditary familial tendency. The gene responsible for its multiple inherited variant is interrelated to chromosome $1 \mathrm{p} 21-$ 22.3 mutation, transmitted in an autosomal dominant mode with incomplete penetrance and $10 \%$ occurrence. ${ }^{7}$ Neurofibromatosis, a condition arising secondary to tumor suppressor gene NF1 mutations, is associated with glomus tumors. Germ line 

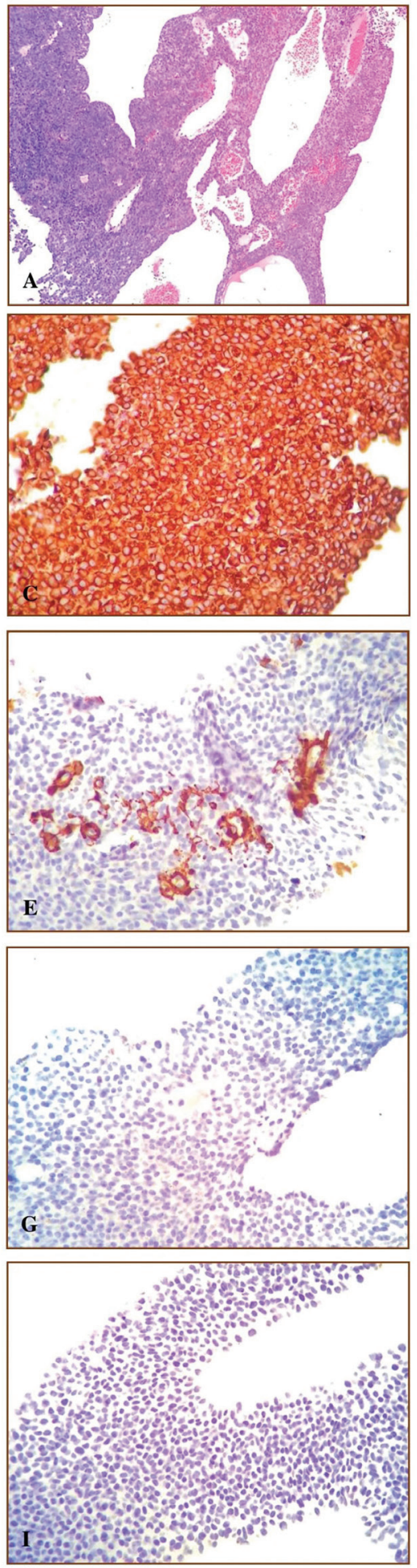
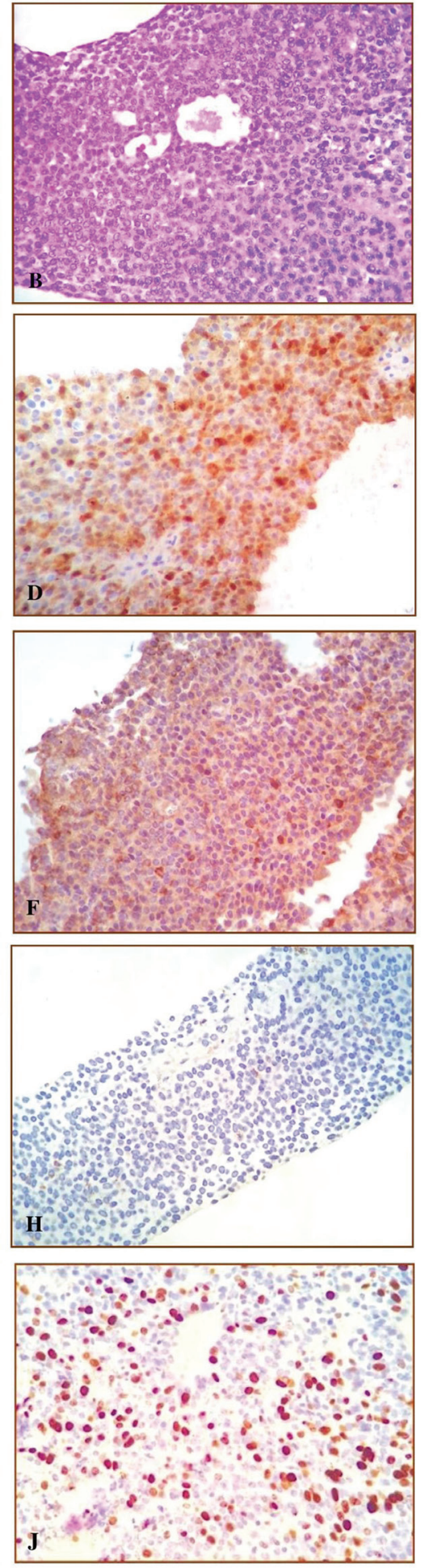

Figure 3. (A) Microscopic sections reveal a lesion composed of neoplastic cells in sheets surrounding endothelial-cell-lined vascular channels supported by a vascular stroma (H\&E 100X). (B) The individual neoplastic cells exhibit polygonal, hyperchromatic to vesicular nuclei, prominent nucleoli and lightly eosinophilic cytoplasm (H\&E 400X). (C) The lesional cells show diffuse cytoplasmic reactivity against Vimentin (400X). (D) and focal reactivity against S100 (400X). (E) The lesional cells show focal reactivity against SMA, prominent on the blood vessels, (400X). (F) and focal reactivity against pancytokeratin (400X). (G) The lesional cells show non-reactivity against Calretinin, (400X). (H) and non-reactivity against Chromogranin (400X). (I) The lesional cells show non-reactivity against Desmin, (400X). (J) and a proliferation fraction of $20-25 \%$ by Ki-67 (400X). mutations in NF1 were found to be present among patients with glomus tumors. On the other hand, somatic NF1 mutations were classified in glomus cells that are alpha-SMA-positive. ${ }^{13}$

The clinical presentation involved in cardiac tumors is usually divided into cardiac symptoms (dyspnea, palpitations, angina, arrhythmias, and cyanosis), systemic manifestations (fever, cachexia, arthralgia, Raynaud's phenomenon, rash, and anemia), and embolic manifestations. ${ }^{14}$ Our patient suffered from two episodes of stroke a year apart. On CT, acute infarct involving the right thalamus, and right hippocampus is seen. Old hemorrhagic infarcts involve the left frontal lobe and bilateral occipital lobes. According to Dias et al, primary cardiac tumors should be considered as a possible cause for embolic manifestations. In his study, cardiac myxoma was the most common cause at $72.6 \%$, followed by fibromas at $6.9 \%$, thrombi at $6.4 \%$, and lastly sarcomas at $6.4 \% .{ }^{14}$ Surgical resection of cardiac tumors remains the treatment of choice in symptomatic cases. ${ }^{15}$ Metastasis is rare but tumor recurrence has been reported. ${ }^{11}$

A differential diagnosis of cardiac glomus tumor include neuroendocrine tumor, mesothelial/monocytic incidental cardiac excresences (MICE) or lesion of aggregated monocytes and mesothelial cells (LAMM), and smooth muscle neoplasms. 
Neuroendocrine tumors, particularly carcinoid tumors, may be confused with glomus tumors, as they are histomorphologically similar, however, the immunohistochemical profile of carcinoid tumors show reactivity to neuroendocrine markers such as chromogranin, as well as with pancytokeratin. ${ }^{3}$ Our case is focally reactive against pancytokeratin but nonreactive against chromogranin. MICE or LAMM are composed of cells with round to oval nuclei rimmed by pink cytoplasm, with prominent nuclear grooves, and occasional nucleoli. These cells are reactive against calretinin, and pancytokeratin. Our case is nonreactive to calretinin. ${ }^{16}$ Smooth muscle neoplasms are composed mainly of spindle cells arranged in fascicles. Immunohistochemically, they are reactive against smooth muscle markers, ${ }^{3}$ such as desmin and smooth muscle actin, which are both nonreactive in our case.

\section{CONCLUSION}

In summary, we report a rare case of a malignant glomus tumor of the heart. This tumor fulfills the criteria for malignancy due to its deep seated location, size $(>2 \mathrm{~cm})$ and proliferation fraction of $20-25 \%$. Clinically, as with any cardiac masses, this may present with a variety of cardiac-related symptoms and may be causes for embolism and stroke. Surgical excision is an effective treatment in symptomatic cases of malignant glomus tumor of the heart. These tumors rarely metastasize and generally follow a benign clinical course despite its classification. However, recurrence has been reported. We further find that although these neoplasms histologically resemble neuroendocrine tumors, MICE/LAMM, and smooth muscle neoplasms, proper histomorphologic analysis and corresponding immunohistochemical tests may be of use in the differentiation of these lesions. To the best of the authors' knowledge this is the first case of malignant glomus tumor of the heart in this institution.

\section{STATEMENT OF AUTHORSHIP}

All authors certified fulfillment of ICMJE authorship criteria.

\section{AUTHOR DISCLOSURE}

The authors declared no conflict of interest.

\section{FUNDING SOURCE}

None.

\section{REFERENCES}

1. Gombos Z, Zhang PJ. Glomus tumor. Arch Path Lab Med. 2008;132(9):1448-52. PMID: 18788860.

2. De Chiara A, Apice G, Mori S, et al. Malignant glomus tumour: a case report and review of the literature. Sarcoma, 2003;7(2):87-91. PMCID: PMC2395518. https://doi. org/10.1080/1357714031000081207.
3. Arïzumi Y, Koizumi H, Hoshikawa M, et al. A primary pulmonary glomus tumor: A case report and review of literature. Case reports in pathology. 2012;2012. Article ID 782304. https://doi.org/10.1155/2012/782304.

4. Jang SH, Cho HD, Lee JH, et al. Mediastinal glomus tumor: a case report and literature review. J Pathol Transl Med. 2015;49(6):520-4. PMID: 26265686. PMCID: PMC4696525. https://doi.org/10.4132/jptm.2015.07.02.

5. Kang G, Park HJ, Kim JY, et al. Glomus tumor of the stomach: a clinicopathologic analysis of 10 cases and review of literature. Gut Liver. 2012;6(1):52-7. PMID: 22375171. PMCID: PMC3286739. https://doi.org/10.5009/gnl.2012.6.1.52.

6. Katz VL, Askin FB, Bosch BD. Glomus tumor of the vulva: a case report. Obstet Gynecol. 1986;67(3 Suppl): 43S-5. PMID: 3003639.

7. Dagur G, Warren K, Miao Y, Singh N, Suh Y, Khan SA. Unusual glomus tumor of the penis. Curr Urol. 2016;9(3):113-8. PMID: 27867327. PMCID: PMC5109964. https://doi.org/10.1159/000442864.

8. StanglR. Glomus tumors in the eye region. BerZusammenkunft Dtsch Ophthalmol Ges. 1977;74:884-6. PMID: 195570.

9. Karapinar K, Kaplan S, Zengin NI, Yucel E. Glomus tumor of the heart: report of an extreme case. Chirurgia. 2005;18(3):125-8.

10. Riesner K, Böcker W. [Cardiac glomus tumor. Light- and electron microscopic investigations (author's transl)]. Z Krebsforsch Klin Onkol Cancer Res Clin Oncol. 1975;84(1):5966. PMID: 171867.

11. Elkrinawi R, Usta E, Baumbach H, Franke UF. Late recurrence of a cardiac glomus tumor. Thorac Cardiovasc Surg. 2012;60(4):305-6. PMID: 21452115. https://doi. org/10.1055/s-0030-1270899.

12. Folpe AL, Fanburg-Smith JC, Miettinen M, Weiss SW. Atypical and malignant glomus tumors: analysis of 52 cases, with a proposal for the reclassification of glomus tumors. Am J Surg Path. 2001;25(1):1-12. PMID: 11145243.

13. Brems H, Park C, Maertens O, Pemov A, Messiaen L, et al. Glomus tumors in neurofibromatosis type 1: genetic, functional, and clinical evidence of a novel association. Cancer Res. 2009;69:(18):7393-401. PMID: 19738042. PMCID: PMC2747722. https://doi.org/10.1158/00085472.CAN-09-1752.

14. Dias RR, Fernandes F, Ramires FJ, Mady C, Albuquerque CP, Jatene FB. Mortality and embolic potential of cardiac tumors. Arq Bras Cardiol. 2014;103(1):13-8. PMID: 25029470. PMCID: PMC4126756.

15. Hoffmeier A, Sindermann JR, Scheld HH, Martens S. Cardiac tumors-diagnosis and surgical treatment. Dtsch Arztebl Int. 2014;111(12):205-11. PMCID: PMC3983698. https://doi.org/10.3238/arztebl.2014.0205.

16. Miller DV, Tazelaar HD. Cardiovascular pseudoneoplasms. Arch Pathol Lab Med. 2010;134(3):362-8. PMID: 20196664. https://doi.org/10.1043/1543-2165-134.3.362.

\footnotetext{
Disclaimer: This journal is OPEN ACCESS, providing immediate access to its content on the principle that making research freely available to the public supports a greater global exchange of knowledge. As a requirement for submission to the PJP, all authors have accomplished an AUTHOR FORM, which declares that the ICMJE criteria for authorship have been met by each author listed, that the article represents original material, has not been published, accepted for publication in other journals, or concurrently submitted to other journals, and that all funding and conflicts of interest have been declared. Consent forms have been secured for the publication of information about patients or cases; otherwise, authors have declared that all means have been exhausted for securing consent.
} 